\title{
BMJ Open Study protocol of a systematic review and qualitative evidence synthesis using two different approaches: Healthcare related needs and desires of older people with post-stroke aphasia
}

\author{
Nadine Janis Pohontsch (D) , ${ }^{1}$ Thorsten Meyer, ${ }^{2}$ Yvonne Eisenmann, ${ }^{3}$ \\ Maria-Inti Metzendorf, ${ }^{4}$ Verena Leve, ${ }^{4}$ Veronika Lentsch, ${ }^{5}$ for the working group \\ 'Qualitative Methods in Health Services Research' of the German Network of \\ Health Services Research
}

To cite: Pohontsch NJ, Meyer T, Eisenmann $\mathrm{Y}$, et al. Study protocol of a systematic review and qualitative evidence synthesis using two different approaches: Healthcare related needs and desires of older people with poststroke aphasia. BMJ Open 2021;11:e039348. doi:10.1136/ bmjopen-2020-039348

- Prepublication history and additional materials for this paper are available online. To view these files, please visit the journal online (http://dx.doi. org/10.1136/bmjopen-2020039348).

Received 12 April 2020 Revised 05 March 2021 Accepted 17 March 2021

Check for updates

(C) Author(s) (or their employer(s)) 2021. Re-use permitted under CC BY-NC. No commercial re-use. See rights and permissions. Published by BMJ.

For numbered affiliations see end of article.

Correspondence to Nadine Janis Pohontsch; n.pohontsch@uke.de

\section{ABSTRACT}

Introduction Stroke is a frequent disease in the older population of Western Europe with aphasia as a common consequence. Aphasia is known to impede targeting treatment to individual patients' needs and therefore may reduce treatment success. In Germany, the postacute care of patients who had stroke is provided by different healthcare institutions of different sectors (rehabilitation, nursing and primary care) with substantial difficulties to coordinate services. We will conduct two qualitative evidence syntheses (QESs) aiming at exploring distinct healthcare needs and desires of older people living with poststroke aphasia. We thereby hope to support the development of integrated care models based on needs of patients who are very restricted to communicate them. Since various methods of QESs exist, the aim of the study embedding the two QESs was to determine if findings differ according to the approach used.

Methods and analysis We will conduct two QESs by using metaethnography (ME) and thematic synthesis (ThS) independently to synthesise the findings of primary qualitative studies. The main differences between these two methods are the underlying epistemologies (idealism (ME) vs realism (ThS)) and the type of research question (emerging (ME) vs fixed (ThS)).

We will search seven bibliographical databases. Inclusion criteria comprise: patients with poststroke aphasia, aged 65 years and older, studies in German/ English, all types of qualitative studies concerning needs and desires related to healthcare or the healthcare system. The protocol was registered in the International Prospective Register of Systematic Reviews, follows Preferred Reporting Items for Systematic Review and Meta-Analysis Protocols guidelines and includes three items from the Enhancing Transparency in Reporting the synthesis of Qualitative Research checklist.

Ethics and dissemination Ethical approval is not required. Findings will be published in a peer-reviewed journal and presented on national conferences.
Strengths and limitations of this study

- We will conduct the qualitative evidence synthesis (QES) in an interdisciplinary research team with members highly experienced in qualitative research.

- All steps of the research process will be discussed both within our research team and with the interdisciplinary working group 'Qualitative Methods in Health Services Research' of the German Network of Health Services Research.

- Study screening and data extraction will be conducted independently in two teams of two authors, with a fifth author mediating any disagreements.

- The conduction of two parallel syntheses will strengthen the validity of the findings and add to the methodological discussion on QES.

- We will only consider publications in English and German.

\section{INTRODUCTION}

Stroke is a frequent disease in Western European countries and the second most common cause of death in Germany and worldwide. ${ }^{1}$ However, in Germany, epidemiological data on stroke rely on estimates derived from stroke registries or data from health insurance companies and therefore vary due to methodological differences. ${ }^{2}$ Probably 243 000-260 000 persons per year are affected by stroke, ${ }^{2}$ with a clear tendency to risk increasing with age, especially for people aged 65 years and older. $^{3}$

Aphasia is known to be a very common consequence of stroke: it occurs in about $30 \%$ of people with stroke. ${ }^{4}$ There are no primary epidemiological data of prevalence and incidence of poststroke aphasia in Germany available. According to Huber 
et $a l^{5}$ in Germany each year, about 25,000 people are newly affected by aphasia after stroke. Aphasic symptoms disappear frequently in the short term, but remain in approximately $56 \%$ of patients more than 10 months after stroke. ${ }^{6}$ This has considerable impact on postacute treatment of stroke with interaction between patient and healthcare provider being affected by patients' communicative deficits. These deficits are even more important in a highly segregated care system with different logics and payment structures.

While the merits of acute care for stroke are known, little is known about postacute healthcare in patients who had stroke. ${ }^{7}$ The coordination of care and the transition between sectors prove to be very challenging. ${ }^{8}$ These transitions are prone to overburden patients, especially patients with aphasia, who literally have a problem to raise their voices. There is a substantial need for integrated care and to inform respective healthcare domains, especially rehabilitation, nursing and primary healthcare services.

Quantitative studies of the needs of survivors of stroke $e^{9-16}$ have revealed a substantial proportion of unmet healthcare needs ranging from at least $51 \%^{14}$ up to $100 \% .^{12} 13$ These studies clearly show that existing unmet needs are unfavourably related to the severity of disease or disability as well as to psychosocial aspects. For example, Andrew et $a l^{10}$ showed that unfulfilled needs were associated with greater disability, fatigue and memory problems. Furthermore, data showed a relation to social status, as the risk for having unmet needs was associated with living in deprived areas, low income or being a member of an ethnic minority group. Chen et $\mathrm{l}^{17}$ discovered that on average, $73.8 \%$ of survivors of stroke survivors have at least one unmet need. The types of needs mentioned in their systematic review ranged from needs related to body functions, activities and participation or environment to service needs in information, transport, home support/ personal care and therapy.

We found three qualitative evidence syntheses (QESs) related to our research question ${ }^{18-20}$ : Hilari et $a l^{18}$ aimed at identifying prognostic factors of health-related quality of life of people with poststroke aphasia, but not on their needs related to healthcare. Moreover, they included only three qualitative studies in their narrative synthesis. In their thematic analysis, Manning et $a l^{19}$ did not focus on healthcare needs or healthcare experiences, but were interested more generally in factors contributing to personal recovery and living successfully with aphasia. The metaethnography (ME) by Pindus $e t a l^{20}$ focused on primary and community care and included all patients who had stroke independent of existing aphasia. Therefore, our review will contribute to answer the question of how health services in Germany could be better suited to the special needs and desires of older people with poststroke aphasia.
Methods for synthesising qualitative research: which one to choose when?

Our aim was not only to generate findings with respect to the needs of people with poststroke aphasia but also to contribute to the methodological discussion on QES. Although theory-based recommendations for deciding which type of QES to choose for certain qualitative research questions exist, ${ }^{21-23}$ to date, there is no empirical evidence showing in which way and to what extent different methods of synthesising lead to different challenges and discrepancies during the review process as well as in the findings.

Of the QES methods presented in Booth et $a l,{ }^{21}$ we chose $\mathrm{ME}^{24}$ and thematic synthesis (ThS $)^{25}$ because of their ontological positions: ME for epistemological idealism and ThS for epistemological realism. In the spectrum between idealism and realism, both methods are closely related ${ }^{23}: \mathrm{ME}$ as representative of objective idealism, that means seeing the world as a result of collectively shared understandings and ThS as a representative of critical realism which assumes that our knowledge of the 'objective' reality is influenced by our perceptions and beliefs. ${ }^{23}{ }^{26}$ Although from different points of viewone that assumes there is no reality 'per se' but peoples' shared experience creates reality, and one that assumes there is one reality, but its awareness is violated by our attitudes, abilities and experiences-the common ground of both may be the idea of people negotiating what reality is.

With regard to the research question, we chose ME as an approach permitting adjustment of the research question in the course of the review conduction and $\mathrm{ThS}$ as an example for a method requiring a fixed research question. ${ }^{21}$

Although different in terms of epistemology and research question, both methods are suited to go beyond the findings of primary studies. This is well known to be the case for ME, ${ }^{24}{ }^{27} 28$ but also confirmed for ThS by Thomas and Harden ${ }^{25}$ or Flemming et al, ${ }^{29}$ who pointed out that ThS findings may as well generate new interpretations, constructs or hypotheses. The possibility to do so mainly relies on the quality and depth of the primary studies. $^{29}$

\section{Description of the two methods chosen for synthesising the primary studies \\ Metaethnography}

Noblit and Hare developed ME as a method to reach a higher level of analysis than one can expect from single studies and that may lead to new research questions. ${ }^{27}$ They defined synthesis as an activity in which separate parts are brought together to form a 'whole' instead of a mere aggregation of data. This construction of the whole is characterised by some degree of innovation, so that the result is greater than the sum of its parts. ${ }^{24}$

ME comprises seven phases: in the first phase, the interest of the research has to be determined. Afterwards it has to be decided which research is relevant to the determined interest (phase II) and the selected studies 
will be read (phase III). This phase is followed by determining how the studies are related (phase IV). According to Noblit and Hare, three different ways of relation are possible: (1) the studies are roughly similar; (2) the studies are contradictory; and (3) the studies build a line of argument. ${ }^{24}$ According to their relation the studies are translated into one another (phase V). The roughly similar studies will be referred to the reciprocal translation, which means that constructs, metaphors and categories from similar studies are translated into each other. If the studies are contradictory, a refutational synthesis will be performed in which contradictory or conflicting findings are investigated, aiming to explain the differences between the single studies. ${ }^{23} 2427$ In phase VI, the translations are synthesised. This procedure should result in an overarching system of so-called third-order categories that means the interpretation of the reviewer. ${ }^{30}$ Additionally, in this phase, a line-of-argument (LOA) synthesis is done. This type of synthesis goes further than translation and permits a wider view on the topic. Although Noblit and Hare ${ }^{24}$ describe the LOA synthesis as one of three possible methods to be used in phase IV, we agree with France $e t a l^{28}$ that it should belong to the interpretive synthesis process. Phase VII relies on reflections about where and in which form to publish the synthesis. Noblit and Hare point out that the publication should be prepared according to the intended audience and the findings achieved. ${ }^{24}$

\section{Thematic synthesis}

$\mathrm{ThS}$ is a method derived from thematic analysis and was developed by Thomas and Harden in the context of health promotion and public health research to address questions about intervention need, appropriateness and acceptability, and factors influencing intervention implementation. This approach shares some characteristics with ME, for example, in the reciprocal translation of descriptive and analytical themes. Moreover, there are similarities with grounded theory as the approach is inductive and themes are developed using a 'constant comparison' method. ${ }^{25}$ The synthesis process consists of three steps: (1) 'line-by-line' coding, (2) developing descriptive themes and (3) generating analytical themes in the last step. ${ }^{25}$

The first step is coding the whole text of the results/ findings section of the primary study line-by-line and thereby translating concepts from one study to another. In step 2, new codes are created to capture the meaning of groups of initial codes (generation of descriptive themes). A synthesis of findings will be generated based on the findings of all original studies and organised by these descriptive themes. During discussion of findings' consistency, inter-relations between codes and descriptive themes as well as a potential hierarchical order will be examined. The last step is the development of analytical themes, which is seen as an interpretative synthesis that goes beyond the content of the original studies. In this stage, models or frameworks may be developed to identify and display relationships between the descriptive themes. $^{25}$

\section{OBJECTIVES}

This protocol is part of a broader (comprehensive) research project addressing two questions: first, which needs and desires in the context of postacute care do people with poststroke aphasia have? To answer this question, we undertake a systematic review of the existing qualitative literature using two different approaches. This part is described here in detail. Beyond that, a second objective of our research was to figure out if, and to what extent, the processes and findings differ according to the respective approach. The details of the procedures required to answer this question are going to be described in a forthcoming article.

\section{METHODS}

The protocol was registered under CRD42020171432 with the International Prospective Register of Systematic Reviews (PROSPERO). Changes will be documented in PROSPERO, providing date and rationale for each modification.

The reporting of this protocol is in accordance with the Preferred Reporting Items for Systematic Reviews and Meta-Analyses Statement for Protocols ${ }^{31}$ and three additional items (items 17-19) from the Enhancing Transparency in Reporting the synthesis of Qualitative Research (ENTREQ) checklist. ${ }^{32}$ The full reports of the reviews will follow the Preferred Reporting Items for Systematic Reviews and Meta-Analyses (PRISMA) ${ }^{33}$ and ENTREQ recommendations, the eMERGe guidance (Meta-Ethnography Reporting Guidance for the ME) ${ }^{34}$ and partly the EPOC (Effective Practice and Organisation of Care) criteria. ${ }^{35}$ Moreover, we will use the GRADECERQual series to guide us through the procedure of assessing confidence in the evidence from the two reviews. ${ }^{36-40}$

\section{Patient and public involvement \\ No patient was involved.}

\section{Search for similar review projects}

To verify that our QES are embedded in current research, we searched for published and unpublished QES concerning our topic. We searched Medline via PubMed for published and PROSPERO for unpublished QES and found three reviews ${ }^{18-20}$ (see the Introduction section for further details).

\section{PICoS (Population, phenomenon of Interest, Context, Study design)}

We chose the PICo tool ${ }^{41}$ to formulate our research question. PICo in qualitative research means population, phenomenon of interest, context. Our objective was to identify studies that explore healthcare needs and desires (phenomenon of interest) of people with poststroke 
Table 1 Inclusion and exclusion criteria for study selection

\section{Inclusion criteria}

\begin{tabular}{ll}
\hline Population & $\begin{array}{l}\text { Adult people, aged } 65 \text { years and older, with } \\
\text { poststroke aphasia }\end{array}$ \\
\hline Interest of the study & Needs and desires related to (the) healthcar
\end{tabular}

(system)

\section{Exclusion criteria}

Study includes only members of particular (minority) groups

Study focuses on special needs related to technical devices

Studies examining certain therapeutic means

\begin{tabular}{|c|c|}
\hline Context of the study & Inpatient or outpatient setting, postacute treatment \\
\hline Study design & $\begin{array}{l}\text { All types of primary qualitative studies (=no reviews), Single-case studies } \\
\text { mixed method studies only if sufficient qualitative } \\
\text { data are provided (eg, separate qualitative data } \\
\text { analysis) }\end{array}$ \\
\hline Languages & German, English \\
\hline Publication status & Published or unpublished, e-first, full text available \\
\hline
\end{tabular}

aphasia (population) during postacute treatment (context). We used the PICo scheme complemented by requirements for the Study design to guide search term selection and inclusion criteria (so-called PICoS scheme; see also table 1).

\section{Search}

\section{Databases and search terms}

Search strategies aimed at identifying qualitative research were developed by an experienced Cochrane Information Specialist using an approach guided by relevant literature. First, a preliminary search strategy guided by the PICoS scheme using text words, and controlled vocabulary (Medical Subject Headings) was designed for MEDLINE. Second, 12 relevant articles meeting the inclusion criteria were identified by conducting and screening a scoping search (see online supplemental file 1). These articles were subsequently used to test and refine the MEDLINE strategy. The test revealed that the concept 'stroke' is not consistently mentioned in the abstracts of relevant publications. Therefore, the concept was omitted from the search strategy to achieve the highest possible sensitivity. The resulting search strategy consists of a search block for 'aphasia' and is combined with validated and sensitive search filters to identify qualitative research, where available for the respective database. The refined MEDLINE strategy was adapted to PsycINFO, Psyindex, CINAHL, Web of Science (Social Science Citation Index), ASSIA and a database indexing grey literature: BASE. Online supplemental file 1 provides details of all search strategies.

\section{Additional search strategies}

In addition to the database search, the review authors will screen the reference lists of included studies.

\section{Inclusion and exclusion criteria}

We will use the criteria outlined in table 1 to select appropriate studies. As healthcare needs differ according to age $^{42}$ and most people affected by aphasia after stroke are of advanced age, ${ }^{43}$ our study focuses on people aged 65 years and older.

\section{Selection of relevant studies}

A PRISMA compliant flowchart ${ }^{33}$ will be used to document and summarise the study selection process. Search results will be uploaded to RAYYAN, ${ }^{44}$ a free software tool allowing reviewer teams to collaborate on screening references online. The selection of relevant studies will be performed in groups of at least two reviewers and will comprise the steps listed in table 2: parallel to the identification of content-related inclusion and exclusion criteria, method-specific inclusion and exclusion criteria will be gathered from the methodological literature (step 1). Content-related criteria are used to screen the search results and establish a list of preliminary included studies (step 2). The full text of the studies will be read in order to remove studies not fulfilling the content-related inclusion criteria in detail (step 3). The resulting sample from this step will be examined using the method-specific criteria (step 4). Neither of the review authors will be blind to the journal titles or to the study authors or the institutions.

\section{Critical appraisal of study quality}

Noblit and Hare ${ }^{24}$ did not take a stance on quality appraisal of the primary studies included in ME. Other authors of metaethnographic methodological research present contrasting opinions on quality appraisal: whereas Atkins et $a l,{ }^{27}$ Campbell $e t a l,{ }^{45}$ Froud et $a l,{ }^{46}$ Pound $e$ e $a l^{47}$ or Toye et $a t^{48}$ seem to be willing to explore the methodological quality of studies to be included in metaethnographies, Britten et $a l^{30}$ or France et $a l^{28}$ point out that time could be spent more efficiently by concentrating on the topic of the review ${ }^{30}$ or that critical appraisal is redundant as long as it has no implications for inclusion or exclusion of studies. ${ }^{28}$ Thomas and Harden performed critical appraisal of the primary studies included in their ThS and undertook a sensitivity analysis to determine the impact of low-quality studies on the findings of the review. ${ }^{25}$ Overall, 
Table 2 Selection process (modified according to Moher et $a l^{33}$ )

1. Identification. Objective: to build a consensus-based framework of content-related inclusion and exclusion criteria and gathering method-specific criteria

Method: Application and refining of content-related in- and exclusion criteria, formulation of method-specific in- and exclusion criteria

Material: titles and abstracts of a random sample of 100 articles for content-related and methodological literature for method-specific criteria

- A subset of 100 articles randomly selected out of the search hits, will be uploaded to RAYYAN.

- Each reviewer screens all of the 100 articles.

- All reviewers compare their results.

- Disagreeing judgements will be discussed and inclusion criteria will be refined through this process.

- Pairs of researchers (VLev and YE, VLen and NP) derive method-specific inclusion and exclusion criteria from the methodological literature.

- The consensus process and the modification of content-related and method-specific inclusion/exclusion criteria (if done) will be documented.

2. Screening. Objective: consensus-based list of preliminary included studies based on cotent-related criteria

Method: screening of all records using content-related inclusion and exclusion criteria

Material: abstracts of all articles retrieved

All hits are screened and evaluated on the basis of the finally consented content-related criteria.

The procedure will be done by two pairs of two reviewers (VLev and YE, VLen and NP). Each pair evaluates half of the hits.

- The respective pair of reviewers will meet to discuss and compare their lists of preliminary included studies.

- If no consensus is reached, a third reviewer (TM) will read the abstract and moderate the consensus process.

- The two lists of preliminary studies (one list per pair) will be joined to one.

- If modifications of content-related inclusion/exclusion criteria are necessary, this will be done and will be documented.

3. Eligibility. Objective: consensus-based list of finally included studies based on content-related criteria, list of excluded studies (including reason for exclusion)

Method: full-text screening of the preliminary included studies using content-related inclusion and exclusion criteria Material: full text

- All reviewers will read the full text of the preliminary included studies.

- Each reviewer creates a list of finally included and excluded studies (with documentation of reason for exclusion).

- Consensus will be sought regarding inclusion and exclusion of studies. The process and decisions will be documented.

- The consensus process will result in one list of finally included and one list of excluded studies (with documentation of reason for exclusion).

4. Inclusion. Objective: method-specific, consensus-based lists of finally included studies, lists of excluded studies for each method (including reason for exclusion)

Method: full-text screening of the studies included in step 3 using method-specific inclusion and exclusion criteria Material: full text

- Method-specific review teams will read the full text of the studies included in step 3.

- Each reviewer creates a list of studies to be included on the basis of method-specific criteria (with documentation of reason for exclusion).

- Consensus about inclusion and exclusion will be sought in the method-specific teams. The process and decisions will be documented.

This process will result in two lists of studies to be included or excluded.

the position of qualitative researchers on this topic is still indecisive. What convinced us to include critical appraisal, were the findings of Pindus et al that a good quality score does not necessarily mean that the study provides sufficient contextual data, ${ }^{20}$ and France $e t a l^{28}$ that poor descriptions of methods do not necessarily equate to a poorly conducted study. Toye $e t a t^{49}$ drew attention to the fact that insightfulness and methodological rigour are different facets of quality and may be quite different. So, we felt that the determination of methodological quality could contribute to the ongoing debate, as long as we go beyond the mere exclusion of methodologically flawed studies. ${ }^{49}$ We will follow Thomas and Harden ${ }^{25}$ and their sensitivity analysis approach and further will explore the relation between insightfulness and methodological quality in the included studies. To determine the methodological quality of the included studies, we chose the widely used Critical Appraisal Skills Programme Checklist for qualitative studies. ${ }^{405152}$

\section{Data extraction and analysis}

For ME as well as for ThS, researcher pairs will independently extract data into spreadsheets and resolve potential discrepancies by discussion. Unsolved disagreements will be discussed with a fifth researcher. From studies employing mixed methods designs, only qualitative data will be extracted. Extracted background data for each study include study design, objective, location, 
setting, participants and method of data collection and analysis.

For the metaethnographic analysis, second-order constructs on subjective needs and desires related to healthcare will be extracted. Second-order constructs are defined as the interpretation of the authors of the primary study. ${ }^{48}$ First-order constructs (the interpretations of the participants of the primary studies) are to be additionally included in our analysis only if clarification of the second-order constructs is required. ${ }^{28}$ The secondorder constructs will lead to third-order constructs: in other words, the interpretation of the reviewer team. ${ }^{25} 30$ For the ThS, all of the text in the results/findings section is to be analysed in three steps: first, a line-by-line coding; second, the organisation of the resulting codes to descriptive themes; and third, the development of analytical themes out of the descriptive ones. These analytical themes correspond to the third-order constructs of the metaethnographical analysis ${ }^{25}$ and therefore can be used to compare the findings of $\mathrm{ME}$ and ThS.

\section{Constant comparison process}

Two independent working groups will each conduct a review using one of the outlined methods. Beyond that, our intention is to compare the challenges, questions and findings arising from each step of the review process to generate empirical material that may clarify the similarities as well as the differences of the two methods. For there is no existing framework that could guide us through the process, we will develop the distinct steps of the comparison during the review process using the method of constant comparison. As an example, we describe how we will proceed if the selection process ends up in two different study samples: to avoid that further differences, particularly the findings of the synthesis in terms of thirdorder constructs/analytical themes, are seen as a result of the divergent study samples, we will analyse both samples with each method. That means: first, each group analyses the studies fulfilling the content-related and the specific inclusion criteria of 'their' method, and afterwards, they include the method-specific studies of the other groupan approach very similar to sensitivity analysis suggested by Thomas and Harden. ${ }^{25}$ This will enable us to distinguish between differences resulting from the methodological steps applied and differences based only on the fact that different studies were included in the QES.

The findings of the two approaches are to be compared with respect to the process of conduction; the resulting third-order constructs (ME)/analytical themes (ThS), the scope of the findings and their potential of generalisation. Because we expect challenges to occur while performing the particular steps of the review, we decided to determine the exact procedures during the process- $-\mathrm{a}$ strategy named 'Gegenstandsangemessenheit' and seen as an indicator of quality in qualitative research. ${ }^{53-55}$ To make this process visible, we will document the respective problem, our discussion and the way we decide to solve it—including the reason for the decision-in a forthcoming methodological article.

A systematic comparison between two qualitative synthesis approaches cannot limit itself to be descriptive but will have to be interpretive to explain the differences. The point of departure of this analysis will be a comparison of the findings of both approaches. Differences and similarities will be identified. In a second step, we will try to understand the differences by looking closer at the respective methods, processes and decisions made during the conduct of the respective study. This will enable us to identify reasons for decisions made and to distinguish between possible reasons that are grounded in the respective methodology or approach versus other reasons, such as personal backgrounds of the reviewers or special theoretical stances. By doing this, we make reference to the comprehensive metastudy approach and plan to explain differences between the findings of the two approaches due to reasons related to data analysis, methods and theories applied..$^{56}$

\section{Author background and reflexivity}

All participating reviewers are experienced qualitative researchers, with different clinical and scientific backgrounds. NP: psychology and health services research, lecturer for qualitative research methods, papers on, for example, qualitative data acquisition and analysis; TM: psychology, rehabilitation sciences and health services research; lecturer in interpretative qualitative methods, papers on, for example, quality criteria of qualitative research and qualitative sampling; VLev/YE: gerontology and palliative care; and VLen: public health, nursing and physical therapy, lecturer for qualitative and health services research.

This diversity in backgrounds will enable us to adopt a comprehensive view on the topic investigated. Constant discussion and regular meetings will help to avoid skewing the analysis and interpretation and thus minimise reviewer bias. Potential threats to credibility as well as reviewer bias will also be minimised by rigorous and transparent discussion in our interdisciplinary research group 'Qualitative Methods in Health Services Research' of the German Network of Health Services Research, as well as determination and documentation of all procedures during the review process. To disclose how our positions may have influenced the review and whether our attitudes toward the research topic changed during the review, we will include a reflexive statement ${ }^{35}$ in the Results section of our review.

\section{DISCUSSION}

By using two rigorous and codified methods (ME and $\mathrm{ThS}$ ) for synthesising qualitative research, we expect to generate in-depth conceptual understanding of what is important for people with poststroke aphasia. The identification of all variations of patients' (living with aphasia) needs and desires related to healthcare may contribute to tailor healthcare in a more patient-centred way-which is 
known to improve therapeutic outcomes. ${ }^{57-59}$ The combination of two different QES methods is unique to our study. As far as we know, such type of review has never been conducted before, and it will allow insights on the impact the QES methods may have not only on the findings but also on the whole review process.

A limitation of our study is that we will not include publications in languages other than German or English. This might limit the range of the findings of our QES.

In addition, a comparison of different QES methods can only be made with some methodological limitations. From a purely conceptual or even idealistic perspective, a strict comparison is hardly possible or only possible for two quite similar approaches. We chose two approaches similar enough to answer the same research question (leaving it open to adapt the question during the course of the study if necessary). We assume, however, that the reality of choice over QES approaches is in practice much more dependent on criteria that go beyond methodological considerations-such as personal background and familiarity with a method and personal preferences of team members. ${ }^{21}$ In practice, therefore, boundaries between different QES approaches might be blurred with regard to concepts, methods or terms. Since there is no ideal comparison in this vein, we find it appropriate to make a few restrictions to the respective QES methodologies in order to allow for substantive comparisons. It is well acknowledged that these restrictions should be an explicit part of the analysis, that is, the approach to comparison.

\section{Ethics and dissemination}

Ethical approval is not required because no human subjects or personal data are directly involved in our review. To ensure dissemination, the findings of the QES will be published in an international peer-reviewed journal and presented on national conferences. A distinct method paper will be published in an international peerreviewed journal.

\section{Author affiliations}

${ }^{1}$ Department of General Practice and Primary Care, University Medical Center Hamburg-Eppendorf, Hamburg, Germany

${ }^{2}$ School of Public Health, Bielefeld University, Bielefeld, Germany

${ }^{3}$ Department of Palliative Medicine, University Hospital of Cologne, Cologne, Germany

${ }^{4}$ Institute of General Practice, Centre for Health and Society, Heinrich-HeineUniversität Düsseldorf, Düsseldorf, Germany

${ }^{5}$ Berufsakademie Nord, University of Cooperative Education, Hamburg, Germany

Contributors NJP is the guarantor. VLen and NJP drafted the manuscript. M-IM designed the search strategy. All authors (NJP, TM, YE, M-IM, VLev and VLen) read, provided feedback on and approved the final manuscript.

Funding The authors have not declared a specific grant for this research from any funding agency in the public, commercial or not-for-profit sectors.

Competing interests None declared.

Patient consent for publication Not required.

Provenance and peer review Not commissioned; externally peer reviewed.

Supplemental material This content has been supplied by the author(s). It has not been vetted by BMJ Publishing Group Limited (BMJ) and may not have been peer-reviewed. Any opinions or recommendations discussed are solely those of the author(s) and are not endorsed by BMJ. BMJ disclaims all liability and responsibility arising from any reliance placed on the content. Where the content includes any translated material, BMJ does not warrant the accuracy and reliability of the translations (including but not limited to local regulations, clinical guidelines, terminology, drug names and drug dosages), and is not responsible for any error and/or omissions arising from translation and adaptation or otherwise.

Open access This is an open access article distributed in accordance with the Creative Commons Attribution Non Commercial (CC BY-NC 4.0) license, which permits others to distribute, remix, adapt, build upon this work non-commercially, and license their derivative works on different terms, provided the original work is properly cited, appropriate credit is given, any changes made indicated, and the use is non-commercial. See: http://creativecommons.org/licenses/by-nc/4.0/.

ORCID iD

Nadine Janis Pohontsch http://orcid.org/0000-0002-0966-4087

\section{REFERENCES}

1 Stahmeyer JT, Stubenrauch S, Geyer S. Häufigkeit und Zeitpunkt von Rezidiven nACh inzidentem Schlaganfall. Dtsch Arztebl International 2019:116:711-7.

2 Kohler M, Deutschbein J, Peschke D, et al. [Stroke occurence in Germany - on the comparability of insurance data and registry-based data]. Fortschr Neurol Psychiatr 2014:82:627-33.

3 Busch MA, Kuhnert R. 12-Monats-Prävalenz von Schlaganfall oder chronischen Beschwerden infolge eines Schlaganfalls in Deutschland. J Health Monit 2017;2:70-6.

4 Engelter ST, Gostynski M, Papa S, et al. Epidemiology of aphasia attributable to first ischemic stroke: incidence, severity, fluency, etiology, and thrombolysis. Stroke 2006;37:1379-84.

5 Huber W, Poeck K, Springer L. Klinik und Rehabilitation der Aphasie: Eine Einführung für Therapeuten, Angehörige und Betroffene ; 26 Tabellen. Stuttgart: Thieme, 2006.

6 Pedersen PM, Jørgensen HS, Nakayama $\mathrm{H}$, et al. Aphasia in acute stroke: incidence, determinants, and recovery. Ann Neurol 1995;38:659-66.

7 Heuschmann P, Busse O, Wagner M, et al. Schlaganfallhäufigkeit und Versorgung von Schlaganfallpatienten in Deutschland. Akt Neurol 2010;37:333-40.

8 Saal S, Kirchner-Heklau U, Müller T. Optimierung Der Überleitung von der stationären in die ambulante Gesundheitsversorgung nACh Schlaganfall (OpTheraS). Rehabilitation 2019;58:39-49.

9 Andrew NE, Kilkenny MF, Lannin NA, et al. Is health-related quality of life between 90 and 180 days following stroke associated with longterm unmet needs? Qual Life Res 2016;25:2053-62.

10 Andrew NE, Kilkenny M, Naylor R, et al. Understanding longterm unmet needs in Australian survivors of stroke. Int $J$ Stroke 2014:9:106-12.

11 Hotter B, Padberg I, Liebenau A, et al. Identifying unmet needs in long-term stroke care using in-depth assessment and the PostStroke Checklist - The Managing Aftercare for Stroke (MAS-I) study. Eur Stroke J 2018;3:237-45.

12 Hughes AK, Woodward AT, Fritz MC, et al. Improving stroke transitions: development and implementation of a social work case management intervention. Soc Work Health Care 2018;57:95-108.

13 Hughes AK, Woodward AT, Fritz MC, et al. Unmet needs of US acute stroke survivors enrolled in a transitional care intervention trial. $J$ Stroke Cerebrovasc Dis 2020;29:104462.

14 McKevitt C, Fudge N, Redfern J, et al. Self-reported long-term needs after stroke. Stroke 2011;42:1398-403.

15 Rothwell K, Boaden R, Bamford D, et al. Feasibility of assessing the needs of stroke patients after six months using the GM-SAT. Clin Rehabil 2013;27:264-71.

16 Ullberg T, Zia E, Petersson J, et al. Perceived unmet rehabilitation needs 1 year after stroke: an observational study from the Swedish stroke register. Stroke 2016;47:539-41.

17 Chen T, Zhang B, Deng Y, et al. Long-term unmet needs after stroke: systematic review of evidence from survey studies. BMJ Open 2019;9:e028137.

18 Hilari K, Needle JJ, Harrison KL. What are the important factors in health-related quality of life for people with aphasia? A systematic review. Arch Phys Med Rehabil 2012;93:S86-95.

19 Manning M, MacFarlane A, Hickey A, et al. Perspectives of people with aphasia post-stroke towards personal recovery and living successfully: a systematic review and thematic synthesis. PLoS One 2019;14:e0214200. 
20 Pindus DM, Mullis R, Lim L. Stroke survivors' and informal caregivers' experiences of primary care and community healthcare services - A systematic review and meta-ethnography. United States, 2018.

21 Booth A, Noyes J, Flemming K. Guidance on choosing qualitative evidence synthesis methods for use in health technology assessments of complex interventions, 2016.

22 Ring N, Ritchie K, Mandava L. A guide to synthesising qualitative research for reserachers undertaking health technology assessments and systematic reviews, 2011.

23 Barnett-Page E, Thomas J. Methods for the synthesis of qualitative research: a critical review. BMC Med Res Methodol 2009;9:59.

24 Noblit GW, Hare RD. Meta-ethnography: synthesizing qualitative studies. 1st edn. Newbury Park, CA: SAGE, 1988.

25 Thomas J, Harden A. Methods for the thematic synthesis of qualitative research in systematic reviews. BMC Med Res Methodol 2008;8:45.

26 Spencer L, Ritchie J, Lewis J. Quality in qualitative evaluation: a framework for assessing research evidence, 2003.

27 Atkins S, Lewin S, Smith H, et al. Conducting a meta-ethnography of qualitative literature: lessons learnt. BMC Med Res Methodol 2008;8:21.

28 France EF, Uny I, Ring N, et al. A methodological systematic review of meta-ethnography conduct to articulate the complex analytical phases. BMC Med Res Methodol 2019;19:35.

29 Flemming K, Booth A, Garside R, et al. Qualitative evidence synthesis for complex interventions and guideline development: clarification of the purpose, designs and relevant methods. BMJ Glob Health 2019;4:e000882.

30 Britten N, Campbell R, Pope C, et al. Using meta ethnography to synthesise qualitative research: a worked example. J Health Serv Res Policy 2002;7:209-15

31 Moher D, Shamseer $L$, Clarke $M$, et al. Preferred reporting items for systematic review and meta-analysis protocols (PRISMA-P) 2015 statement. Syst Rev 2015;4:1.

32 Tong A, Flemming K, Mclnnes E, et al. Enhancing transparency in reporting the synthesis of qualitative research: ENTREQ. BMC Med Res Methodol 2012;12:181

33 Moher D, Liberati A, Tetzlaff J, et al. Preferred reporting items for systematic reviews and meta-analyses: the PRISMA statement. $B M J$ 2009;339:b2535.

34 France EF, Cunningham M, Ring N, et al. Improving reporting of meta-ethnography: the eMERGe reporting guidance. Psychooncology 2019;28:447-58.

35 Glenton C, Bohren MA, Downe S. EPOC qualitative evidence synthesis: protocol and review template, 2019. Available: https:// epoc.cochrane.org/sites/epoc.cochrane.org/files/public/uploads/ Resources-for-authors2017/epoc_qes_protocol_and_review_ template.pdf

36 Glenton C, Carlsen B, Lewin S, et al. Applying GRADE-CERQual to qualitative evidence synthesis findings-paper 5: how to assess adequacy of data. Implement Sci 2018;13:14.

37 Noyes J, Booth A, Lewin S. Applying GRADE-CERQual to qualitative evidence synthesis findings-paper 6: how to assess relevance of the data. Implement Sci 2018;13:4

38 Colvin CJ, Garside R, Wainwright M, et al. Applying GRADECERQual to qualitative evidence synthesis findings-paper 4: how to assess coherence. Implement Sci 2018;13:13.

39 Lewin S, Bohren M, Rashidian A, et al. Applying GRADE-CERQual to qualitative evidence synthesis findings-paper 2: how to make an overall CERQual assessment of confidence and create a summary of qualitative findings table. Implement Sci 2018:13:10

40 Munthe-Kaas H, Bohren MA, Glenton C, et al. Applying GRADECERQual to qualitative evidence synthesis findings-paper 3: how to assess methodological limitations. Implement Sci 2018;13:9.

41 Stern C, Jordan Z, McArthur A. Developing the review question and inclusion criteria. Am J Nurs 2014:114:53-6.

42 Bayer-Pörsch K. Kommunikation und Kooperation in Der Interaktion von Gesundheitssystem und Patienten: Eine Untersuchung am Beispiel Der Behandlung des diabetes mellitus Im Raum Frankfurt/ main dissertation. Gießen 2003.

43 Busch MA, Schienkiewitz A, Nowossadeck E. Prävalenz des Schlaganfalls bei Erwachsenen Im alter von 40 bis 79 Jahren in Deutschland: Ergebnisse Der studie Zur Gesundheit Erwachsener in Deutschland (DEGS1). Bundesgesundheitsblatt Gesundheitsforschung Gesundheitsschutz 2013;56:656-60.

44 Ouzzani M, Hammady H, Fedorowicz Z, et al. Rayyan-a web and mobile APP for systematic reviews. Syst Rev 2016;5:210.

45 Campbell R, Pound P, Pope C. Evaluating meta-ethnography. a synthesis of qualitative reserach on lay experiences of diabetes and diabetes care. Soc Sci Med 2003;65:671-84.

46 Froud R, Patterson S, Eldridge S, et al. A systematic review and meta-synthesis of the impact of low back pain on people's lives. BMC Musculoskelet Disord 2014;15:50.

47 Pound P, Britten N, Morgan M, et al. Resisting medicines: a synthesis of qualitative studies of medicine taking. Soc Sci Med 2005;61:133-55.

48 Toye $\mathrm{F}$, Seers $\mathrm{K}$, Allcock N, et al. Meta-ethnography 25 years on: challenges and insights for synthesising a large number of qualitative studies. BMC Med Res Methodol 2014;14:80.

49 Toye F, Seers K, Allcock N, et al. 'Trying to pin down jelly' - exploring intuitive processes in quality assessment for meta-ethnography. BMC Med Res Methodol 2013;13:46.

50 Carroll C, Booth A. Quality assessment of qualitative evidence for systematic review and synthesis: is it meaningful, and if so, how should it be performed? Res Syn Meth 2015;6:149-54.

51 Noyes J, Booth A, Cargo M, et al. Cochrane qualitative and implementation methods group guidance series-paper 1: introduction. J Clin Epidemiol 2018;97:35-8.

52 France EF, Ring N, Thomas R, et al. A methodological systematic review of what's wrong with meta-ethnography reporting. BMC Med Res Methodol 2014:14:119.

53 Lamnek S. Qualitative Sozialforschung. 5th edn. Weinheim/Basel: Beltz, 2010

54 Flick U. Qualitative Sozialforschung. 5th edn. Reinbek: Rowohlt, 2012.

55 Strübing J, Hirschauer S, Ayaß R, et al. Gütekriterien qualitativer Sozialforschung. ein Diskussionsanstoß. Zeitschrift für Soziologie 2018;47:83-100.

56 Paterson BL, Thorne SE, Canam C. Meta-study of qualitative health research: a practical guide to meta-analysis and meta-synthesis. Thousand Oaks, CA: SAGE, 2001

57 Robinson JH, Callister LC, Berry JA, et al. Patient-centered care and adherence: definitions and applications to improve outcomes. J Am Acad Nurse Pract 2008;20:600-7.

58 Poochikian-Sarkissian S, Sidani S, Ferguson-Pare M, et al. Examining the relationship between patient-centred care and outcomes. Can J Neurosci Nurs 2010;32:14-21.

59 Sidani S. Effects of patient-centered care on patient outcomes: an evaluation. Res Theory Nurs Pract 2008;22:24-37. 